\title{
Parameter selection and longitudinal phase space simulation for a single stage X-band FEL driver at $250 \mathrm{MeV}$
}

\author{
Yipeng Sun* and Tor Raubenheimer, Juhao Wu \\ SLAC, Stanford, CA 94025, USA
}

\begin{abstract}
Hard x-ray Free electron lasers (FEL) are being built or proposed at many accelerator laboratories as it supports wide range of applications in many aspects. Most of the hard x-ray FEL design is similar with the SLAC Linac Coherent Light Source (LCLS), which features a two (or multiple) stage bunch compression. For the first stage of the bunch compression, usually the beam is accelerated in a lower-frequency RF section (such as S-band for LCLS), and then the longitudinal phase space is linearized by a higher-frequency RF section (harmonic RF, such as X-band for LCLS). In this paper, a compact hard x-ray FEL design is proposed, which is based on X-band RF acceleration and eliminating the need of a harmonic RF. The parameter selection and relation is discussed, and the longitudinal phase space simulation is presented.
\end{abstract}

\section{Overview}

Free electron lasers (FEL) was invented by J. Madey and demonstrated for the first time at Stanford University in 1970s [1] [2]. There are several Free electron lasers being constructed or proposed afterwards everywhere in the world [3] [4] [5].

The FEL coherence condition of the electron beam in the undulators requires a large charge density, a small emittance and small energy spread. The RMS electron bunch length from the injector is in the ps scale, with a bunch charge in the range of hundreds $\mathrm{pC}$ to several $\mathrm{nC}$, which means that the current is roughly $0.1 \mathrm{kA}$. According to the requirement from soft x-ray lasing and hard x-ray lasing, a peak current of $1 \mathrm{kA}$ and $3 \mathrm{kA}$ is needed respectively. Thus the bunch has to be compressed. Usually a two stage bunch compression or multipole stage bunch compression is adopted. The z-correlated energy chirp is normally established by letting the beam pass through a section of RF cavities, with a RF phase off crest. As stated above, S-band RF (3 GHz) acceleration could be applied in this section. Due to the nature of RF acceleration wave, the chirp on the bunch is not linear, but has the RF curvature on it. In order to linearize the energy chirp, a harmonic RF section with higher frequency is needed. For LCLS a short $\mathrm{X}$-band $\mathrm{RF}$ section $(12 \mathrm{GHz})$ is used which is a fourth order harmonic.

The linearized bunch is then passing by a dispersive region, in which the particles with different energy have different path length. A four dipole chicane is the natural choice for the dispersive region. As the example illustrated in Figure 1, the head of the bunch has smaller energy, and gets a stronger bending kick from the dipole magnet, then has a longer path length in the dispersive region. Similarly, the tail of the bunch has larger energy and shorter path length in the dispersive region. At the exit of the dispersive region, the relative longitudinal position of the head and tail of the bunch both move to the center of the bunch, so the bunch length will be shorter.

\section{X-band FEL driver}

Another way to do the bunch compression, is to eliminate the harmonic RF section, and replace the four dipole chicane by a dispersive region which has special higher order longitudinal dispersion terms. This option may have the advantage of compact size at same energy, as it only uses X-band acceleration structures which can provide a much

*Electronic address: yisun@slac.stanford.edu 


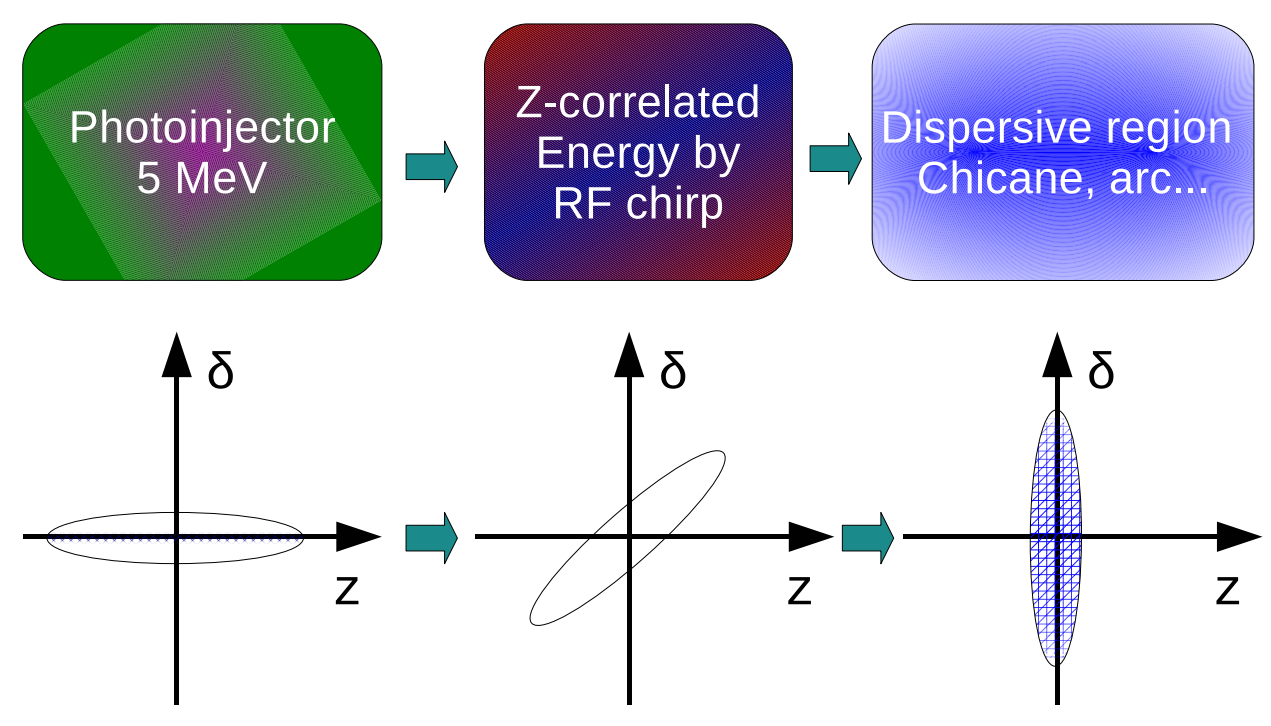

Figure 1: Sketch for magnetic bunch compression.

\section{X-band FEL Driver}

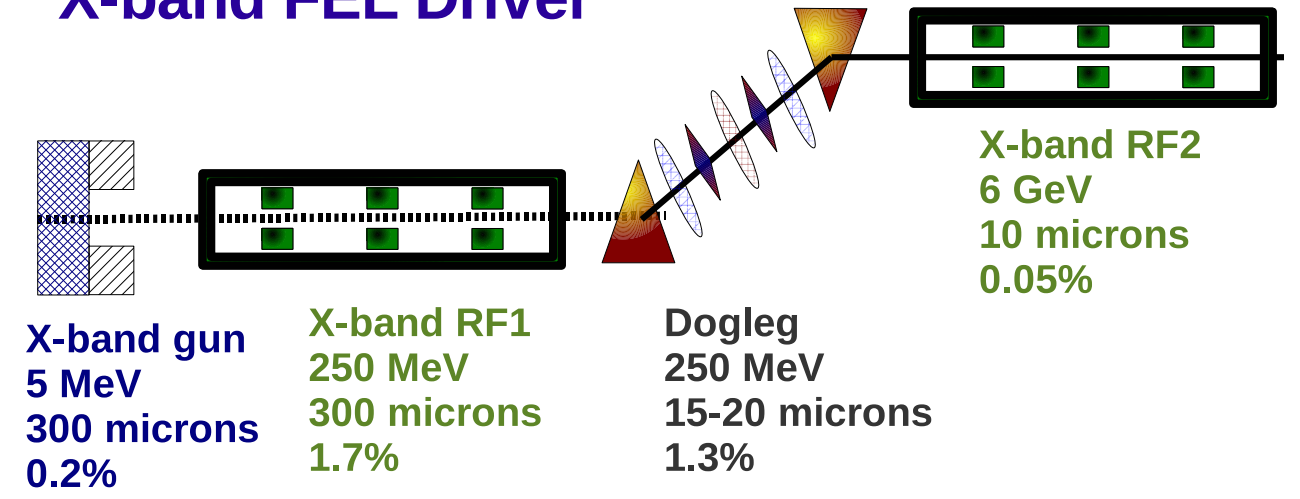

Figure 2: Sketch for an X-band FEL driver.

higher accelerating gradient than S-band. Besides the shorter wavelength of X-band also makes the establishment of the chirp easier, which can then run with a RF phase more close to the crest and save power.

To achieve that condition with normal magnets, one might need to place quadrupoles (or sextupoles) between the dipoles to generate a second order dispersion $T_{566}$ which has a specified relation with the first order dispersion $R_{56}$. It is worth to mention that for a four dipole chicane (or other similar shape with only dipoles and drifts), there is a simple relation between $T_{566}$ and $R_{56}$, which is $T_{566}=-1.5 R_{56}$. To supress the RF curvature on the bunch chirp, one needs a dispersive region with same sign of $R_{56}$ and $T_{566}$. The details are discussed in the following sections.

The basic shape of the X-band FEL driver being proposed here is sketched in Figure 2. The electron beam from 
the photo-injector has a RMS bunch length of $300 \mu \mathrm{m}$, a beam energy of $5 \mathrm{MeV}$ and a RMS energy spread of $10 \mathrm{keV}$. It is then injected off-crest into an X-band RF acceleration section whose length is approximately 3 meters with an average gradient of $80 \mathrm{MV} / \mathrm{m}$. The beam energy is $250 \mathrm{MeV}$ after acceleration and the RMS energy spread is 1.7\%. After passing by a dogleg (or another similar structure) which has a specially designed higher order dispersion, the bunch length is compressed 15-20 times, to roughly $15 \mu \mathrm{m}$. The RMS energy spread is reduced to $1.3 \%$ with momentum collimators placed between the dipoles, and can be further damped to $0.05 \%$ if the final beam energy is accelerated to $6 \mathrm{GeV}$.

\section{Parameter relations for magnetic bunch compression}

As discussed above, the key point of magnetic bunch compression is to firstly establish an energy correlation along the bunch longitudinal axis, then let the beam pass by a dispersive region in which particles with different energy have different path length. In this section, the analytical formulae are derived, to calculate the final bunch length and the required longitudinal dispersion up to third order.

\subsection{RF chirp}

The energy correlation (chirp) is established by RF acceleration off crest. For any one particle in a bunch, its relative energy offset after passing by this RF acceleration can be expressed as

$$
\delta(z)=\delta_{i} \frac{E_{i 0}}{E_{f 0}}+\frac{e V_{0} \cos \left(\phi+k z_{i}\right)}{E_{f 0}}=a \cdot \delta_{i}+\frac{e V_{0} \cos \left(\phi+k z_{i}\right)}{E_{f 0}}
$$

where $\delta_{i}$ denotes the initial un-correlated energy offset, $E_{i 0}$ central energy before RF acceleration, $E_{f 0}$ central energy after RF acceleration, $V_{0}$ the RF voltage, $\phi$ the RF phase, $k=\frac{2 \pi}{\lambda}$ the RF wave number, $\lambda$ the RF wave length and $z_{i}$ particle's longitudinal coordinate relative to the bunch center.

By using the following relation in formula (2), and Taylor series of the trigonometric functions 'Sine' and 'Cosine' as expressed in formulae (3) and (4), one could express the z-correlated energy offset $\delta(z)$ as a polynomial of $z$.

$$
\begin{gathered}
\cos (\theta+\phi)=\cos \theta \cos \phi-\sin \theta \sin \phi \\
\cos x=\sum_{n=0}^{\infty} \frac{(-1)^{n}}{(2 n) !} x^{2 n} \\
\sin x=\sum_{n=0}^{\infty} \frac{(-1)^{n}}{(2 n+1) !} x^{2 n+1}
\end{gathered}
$$

Thus, the z-correlated energy offset can be written as

$$
\delta(z)=a \cdot \delta_{i}+h_{1} z+h_{2} z^{2}+h_{3} z^{3}+\ldots
$$

where $h_{1}$ denotes the first order chirp, $h_{2}$ second order chirp, and $h_{3}$ third order chirp.

One could easily derive that the chirp up to third order can be expressed as in the following formulae, assuming that the initial beam energy is very small and could be negligible $\left(\Delta E \approx E_{f 0}\right)$. 


$$
\begin{gathered}
h_{1}=-\frac{k e V_{0} \sin \phi}{E_{f 0}} \approx-k \tan \phi \\
h_{2}=-\frac{k^{2} e V_{0} \cos \phi}{2 E_{f 0}}=-\frac{k^{2} \Delta E}{2 E_{f 0}} \approx-\frac{k^{2}}{2} \\
h_{3}=\frac{k^{3} e V_{0} \sin \phi}{6 E_{f 0}} \approx \frac{k^{3}}{6} \tan \phi
\end{gathered}
$$

where $e$ denotes the electron charge, $\Delta E$ the energy gain through $\mathrm{RF}$ acceleration.

\subsection{Dispersion region}

The beam then passes by a dispersive region which can be either a dogleg or a chicane plus sextupole, depending on the sign of the chirp. For a bunch with lower energy at the head, one should choose a dispersive region with negative $R_{56}$ (chicane plus sextupole). On the other hand, for a bunch with lower energy at the tail, one should choose positive $R_{56}$ (dogleg, or in detail, dipole plus quadrupoles).

After passing by the dispersive region, the longitudinal coordinate (relative to the bunch center) of any particle can be expressed as

$$
\begin{gathered}
z_{f}(\delta)=z_{i}+b_{1} \delta+b_{2} \delta^{2}+b_{3} \delta^{3}+\ldots \\
z_{f}(\delta)=z_{i}+R_{56} \delta+T_{566} \delta^{2}+U_{5666} \delta^{3}+\ldots
\end{gathered}
$$

where $z_{i}$ denotes the initial longitudinal coordinate relative to the bunch center, $b_{1}$ the first order longitudinal dispersion, $b_{2}$ the second order dispersion, and $b_{3}$ the third order dispersion.

Insert the z-correlated energy offset $\delta(z)$ as expressed in formula (5) into the above equation (up to 3rd order), and neglect the relatively small initial un-correlated energy offset in the higher order terms, one could express the final longitudinal coordinate of any particle as a function of its initial coordinate, as expressed in formula (10).

$$
z_{f}=z_{i}+b_{1}\left(a \cdot \delta_{i}+h_{1} z_{i}+h_{2} z_{i}^{2}+h_{3} z_{i}^{3}\right)+b_{2}\left(h_{1} z_{i}+h_{2} z_{i}{ }^{2}+h_{3} z_{i}^{3}\right)^{2}+b_{3}\left(h_{1} z_{i}+h_{2} z_{i}{ }^{2}+h_{3} z_{i}^{3}\right)^{3}+\ldots
$$

It is observed that the final longitudinal distribution depends on the initial distribution, the energy chirp and the dispersion terms.

\subsection{Dispersion terms of Chicane or wiggler}

For any bunch compressor without quadrupole, such as a four dipole chicane or a wiggler, the total path length can be expressed as (under a small angle approximation)

$$
s=L \sqrt{1+\theta^{2}} \approx L\left(1+\frac{1}{2} \theta^{2}\right)
$$

where $L$ denotes the effective length of the chicane, $\theta$ the effective bending angle of the dipole magnet.

By using the following Taylor series as shown in formula (12), the difference of path length between an offmomentum particle (with energy offset $\delta$ ) and an on-momentum particle is shown in formula (13). 


$$
\begin{gathered}
\frac{x}{(1-x)^{2}}=\sum_{n=1}^{\infty} n x^{n} \\
\Delta s \propto \theta^{2}=\left(\frac{\theta_{0}}{1+\delta}\right)^{2}=\theta_{0}^{2}\left(1-2 \delta+3 \delta^{2}-4 \delta^{3}+\ldots\right)
\end{gathered}
$$

where $\theta_{0}$ denotes the bending angle of the dipole magnet on the on-momentum particle.

The higher order dispersion terms can then be expressed as

$$
\begin{aligned}
& T_{566}=-\frac{3}{2} R_{56} \\
& U_{5666}=2 R_{56}
\end{aligned}
$$

where $R_{56}$ denotes the first order longitudinal dispersion, $T_{566}$ the second order dispersion, and $U_{5666}$ the third order dispersion.

From formulae (14) and (15), it is observed that the second and third order dispersion has a fixed ratio over the first order dispersion, for any bunch compressor with only dipole and drift space.

\subsection{Bunch length after compression}

Assuming the electron bunch preserves a Gaussian distribution after passing by the dispersive region, the RMS bunch length can then be calculated as an integral shown in formula (16).

$$
\sigma_{z}^{2}=\iint z_{f}^{2}(z, \delta) \cdot f(z, \delta) d z d \delta
$$

where $f(z, \delta)$ denotes a Gaussian distribution in both $z$ and $\delta$ as expressed in formula (17).

$$
f(z, \delta)=\frac{1}{2 \pi \sigma_{z} \sigma_{\delta}} \cdot e^{\frac{-z^{2}}{2 \sigma_{z}^{2}}-\frac{\delta^{2}}{2 \sigma_{\delta}^{2}}}
$$

As the integral of all the odd functions is zero, one finds the following is true.

$$
\begin{aligned}
& \iint z_{i} f(z, \delta) d z d \delta=0 \\
& \iint \delta_{i} f(z, \delta) d z d \delta=0 \\
& \iint z_{i} \delta_{i} f(z, \delta) d z d \delta=0 \\
& \iint z_{i}^{3} f(z, \delta) d z d \delta=0
\end{aligned}
$$

Taking advantage of the integral as expressed in formula (22), one can derive the integral as shown in formulae (23)-(25). 


$$
\begin{gathered}
\int_{-\infty}^{\infty} x^{2 n} e^{-x^{2} / g^{2}} d x=2 \sqrt{\pi} \frac{(2 n) !}{n !}\left(\frac{g}{2}\right)^{2 n+1} \\
\sigma_{z_{i}}{ }^{2}=\iint z_{i}{ }^{2} f(z, \delta) d z d \delta \\
3 \cdot \sigma_{z_{i}}{ }^{4}=\iint z_{i}{ }^{4} f(z, \delta) d z d \delta \\
15 \cdot \sigma_{z_{i}}{ }^{6}=\iint z_{i}{ }^{6} f(z, \delta) d z d \delta
\end{gathered}
$$

where $\sigma_{z, i}$ denotes the initial bunch length.

In formula (10), keep the terms up to second order energy chirp and dispersion, one finds that $z_{f}^{2}(z, \delta)$ equals

$$
\begin{array}{r}
z_{f}^{2}\left(z, \delta_{i}\right)=a^{2} R_{56}^{2} \delta_{i}{ }^{2}+2 R_{56} \cdot a \cdot \delta_{i}\left(1+h_{1} R_{56}\right) \cdot z+\left(\left(1+h_{1} R_{56}\right)^{2}+2 R_{56} \cdot a \cdot \delta_{i}\left(h_{2} R_{56}+h_{1}^{2} \cdot T_{566}\right)\right) \cdot z^{2} \\
+(\ldots \ldots) \cdot z^{3} \\
+\left(\left(h_{1}^{2} \cdot T_{566}+h_{2} \cdot R_{56}\right)^{2}+4 h_{1} h_{2} T_{566}\left(1+h_{1} R_{56}\right)+2 h_{2}^{2} R_{56} T_{566} \cdot a \cdot \delta_{i}\right) \cdot z^{4} \\
+(\ldots \ldots) \cdot z^{5}+(\ldots \ldots) \cdot z^{6}+(\ldots \ldots) \cdot z^{7}+(\ldots \ldots) \cdot z^{8}
\end{array}
$$

where $\delta_{i}$ denotes the initial un-correlated energy offset, $z_{f}(z, \delta)$ the final longitudinal coordinate, and $z$ the initial longitudinal coordinate.

Take advantage of formulae (18)-(21), keep up to fourth order terms $\left(z^{4}\right)$, and apply the optimal compression condition as expressed in formula (27), one could derive the final bunch length as shown in formula (28).

$$
\begin{gathered}
1+h_{1} R_{56}=0 \\
h_{1}^{2} \cdot T_{566}+h_{2} \cdot R_{56}=0 \\
h_{3} R_{56}+2 h_{1} h_{2} T_{566}+h_{1}^{3} U_{5666}=0 \\
\sigma_{z}^{2}=a^{2} R_{56}^{2} \sigma_{\delta i}^{2}+\left(1+h_{1} R_{56}\right)^{2} \cdot \sigma_{z, i}{ }^{2}+3 \cdot\left(h_{1}^{2} \cdot T_{566}+h_{2} \cdot R_{56}\right)^{2} \cdot \sigma_{z, i}
\end{gathered}
$$

where $\sigma_{\delta i}$ denotes the initial un-correlated RMS energy spread, $\sigma_{z, i}$ initial bunch length.

At the same time, in formula (10) keep the terms up to third order energy chirp and dispersion, one finds that $z_{f}^{2}(z, \delta)$ equals

$$
\begin{array}{r}
z_{f}^{2}\left(z, \delta_{i}\right)=a^{2} R_{56}^{2} \delta_{i}{ }^{2}+2 R_{56} \cdot a \cdot \delta_{i}\left(1+h_{1} R_{56}\right) \cdot z+\left(\left(1+h_{1} R_{56}\right)^{2}+2 R_{56} \cdot a \cdot \delta_{i}\left(h_{2} R_{56}+h_{1}^{2} \cdot T_{566}\right)\right) \cdot z^{2} \\
+(\ldots \ldots) \cdot z^{3} \\
+\left[\left(h_{1}^{2} \cdot T_{566}+h_{2} \cdot R_{56}\right)^{2}+4 h_{1} h_{2} T_{566}\left(1+h_{1} R_{56}\right)+2 h_{2}^{2} R_{56} T_{566} \cdot a \cdot \delta_{i}+\right. \\
\left.\left(4 h_{1} R_{56} T_{566} \cdot a \cdot \delta_{i}+2 R_{56}\left(1+h_{1} R_{56}\right)\right) \cdot h_{3}+6 h_{1}^{2} h_{2} R_{56} U_{5666} \cdot a \cdot \delta_{i}+2 h_{1}^{3} U_{5666}\left(1+h_{1} R_{56}\right)\right] \cdot z^{4} \\
+(\ldots \ldots) \cdot z^{5} \\
+\left((\ldots) \cdot a \cdot \delta_{i}+(\ldots) \cdot\left(1+h_{1} R_{56}\right)+\left(h_{3} R_{56}+2 h_{1} h_{2} T_{566}+h_{1}^{3} U_{5666}\right)^{2}\right) \cdot z^{6} \\
+(\ldots \ldots) \cdot z^{7}+(\ldots \ldots) \cdot z^{8}+\ldots \ldots+(\ldots \ldots) \cdot z^{18}
\end{array}
$$


where $\delta_{i}$ denotes the initial un-correlated energy offset, $z_{f}(z, \delta)$ the final longitudinal coordinate, and $z$ the initial longitudinal coordinate.

For a chicane (or a wiggler) and a positive RF slope, one finds $R_{56}<0, T_{566}>0, U_{5666}<0, h_{1}>0, h_{2}<0$, $h_{3}>0$. By observing the above formula, one can conclude that the second and third order dispersion $\left(T_{566}\right.$ and $\left.U_{5666}\right)$ always make the final bunch length longer.

\subsection{Linearization by choosing proper $R_{56}, T_{566}$ and $U_{5666}$}

As the scheme applied at the SLAC Linac Coherent Light Source (LCLS) [4], a linear energy chirp could be established with the help of the harmonic RF section. Usually deceleration is applied at the harmonic RF and then a linearized bunch is produced. The details of linearization using harmonic RF is discussed in Appendix.

Here a different approach is investigated, which keeps the RF curvature on the chirp, but uses a specified optics in the bunch compressor to fulfill a linearized bunch compression process. Again, after passing by the dispersive region, the longitudinal coordinate of any particle relative to the bunch center can be expressed as

$$
z_{f}=z_{i}+b_{1}\left(h_{1} z_{i}+h_{2} z_{i}{ }^{2}+h_{3} z_{i}{ }^{3}\right)+b_{2}\left(h_{1} z_{i}+h_{2} z_{i}{ }^{2}+h_{3} z_{i}^{3}\right)^{2}+b_{3}\left(h_{1} z_{i}+h_{2} z_{i}{ }^{2}+h_{3} z_{i}{ }^{3}\right)^{3}+\ldots
$$

Keep up to third order terms, the above formula can be expressed as

$$
z_{f}=a_{1} \cdot z_{i}+a_{2} \cdot z_{i}^{2}+a_{3} \cdot z_{i}^{3}
$$

The coefficient of the 1 st order term $z_{i}$ is

$$
a_{1}=1+b_{1} \cdot h_{1}
$$

The coefficient of the 2 nd order term $z_{i}^{2}$ is

$$
a_{2}=b_{1} \cdot h_{2}+b_{2} \cdot h_{1}^{2}
$$

The coefficient of the 3 rd order term $z_{i}{ }^{3}$ is

$$
a_{3}=b_{1} \cdot h_{3}+b_{2} \cdot 2 h_{1} h_{2}+b_{3} \cdot h_{1}^{3}
$$

Make all three coefficients equal zero, one finds the relation between $R_{56}$ and $R F$ parameters, also the relation between $R_{56}$ and high order dispersion terms $T_{566}$ and $U_{5666}$. That is the solution to achieve a minimum final bunch length, which only depends on the initial un-correlated energy spread and $R_{56}$.

In detail, given the condition that the first order coefficient is zeroed by choosing a proper RF phase and linear dispersion $R_{56}, a_{1}=0$, one finds

$$
R_{56}=b_{1}=-\frac{1}{h_{1}}=\frac{E_{f 0}}{k \Delta E \tan \phi}
$$

With $a_{1}=0$ and an un-correlated initial $\sigma_{\delta i}$, the contribution to $z^{4}$ from all the cross terms is zero, as illustrated in formulae (26) and (29). Under these conditions, by letting $a_{2}=0$ one finds the required $T_{566}$ as shown below.

$$
T_{566}=b_{2}=-\frac{h_{2}}{h_{1}^{2}} \cdot R_{56}=\frac{R_{56}}{2} \cdot \frac{E_{f 0}}{\Delta E} \cdot \frac{1}{\tan \phi^{2}}
$$

Similarly, with $a_{1}=0, a_{2}=0$ and an un-correlated initial $\sigma_{\delta i}$, the contribution to $z^{6}$ from all the cross terms is zero too, as illustrated in formula (29). Under these conditions, by letting $a_{3}=0$ one finds the required $U_{5666}$ as shown below. 


$$
U_{5666}=b_{3}=R_{56} \cdot \frac{E_{f 0}}{\Delta E} \cdot\left(\frac{1}{6 \tan \phi^{2}}+\frac{1}{2 \tan \phi^{4}}\right)
$$

where $\Delta E$ denotes the energy gain through the RF acceleration.

The effectiveness of these formulae derived above are checked and confirmed through LiTrack simulations [7]. An electron bunch with an initial bunch length of $300 \mu \mathrm{m}$ is generated at a beam energy of $5 \mathrm{MeV}$. It then passes through an x-band acceleration section on a RF phase of 13 degree. The beam energy is accelerated up to $250 \mathrm{MeV}$. Finally it is the dispersive region with the proper linear and higher order dispersions. The main parameters are listed in Table 1. As shown in Figure 3, the final bunch length is only dependent on the initial un-correlated energy spread. With an initial energy spread of $0.01 \%$ (case 'c'), the final bunch length is as short as $0.1 \mu m(0.3 f s)$.

Table I: Main parameters used in the simulation.

\begin{tabular}{c|c|c}
\hline Parameter & Unit & Value \\
\hline$\sigma_{z 0}$ & $\mu m$ & 300 \\
$E_{i}$ & $M e V$ & 5 \\
$E_{f}$ & $M e V$ & 250 \\
$\phi_{R F}$ & degree & 13 \\
$f_{R F}$ & $G H z$ & 12 \\
$R_{56}$ & $m$ & 0.01757 \\
$T_{566}$ & $m$ & 0.165 \\
\hline
\end{tabular}

\subsection{RMS energy spread after compression}

Similar as the expression for final bunch length, the final energy spread can be calculated as the following integral.

$$
\sigma_{\delta}^{2}=\iint \delta_{f}^{2}(z, \delta) \cdot f(z, \delta) d z d \delta
$$

where $f(z, \delta)$ denotes a Gaussian distribution in both $z$ and $\delta$ as expressed in formula (17).

Assuming un-correlated initial $z$ and $\delta$ for any particle in a bunch, one finds that the energy spread after compression can be expressed as (keep up to 1st order term)

$$
\sigma_{\delta}^{2}=a^{2} \sigma_{\delta i}^{2}+h_{1}^{2} \cdot \sigma_{z, i}^{2}
$$

where $a=\frac{E_{i 0}}{E_{f 0}}$ denotes the energy ratio, $h_{1}$ the first order RF chirp.

For most of the case, the term $a^{2} \sigma_{\delta i}^{2}$ which is from initial energy spread tends to be small. And the dominant term in the final energy spread would be from the linear chirp plus initial bunch length, which is known as the 'intrinsic' energy spread.

Keep up to third order terms and apply approximation, one finds the energy offset of any particle can be expressed as in formula (40).

$$
\delta_{f}^{2}=\left(a \cdot \delta_{i}\right)^{2}+h_{1}^{2} z^{2}+2 h_{1} h_{2} z^{3}+\left(2 h_{1} h_{3}+h_{2}^{2}\right) z^{4}+2 h_{2} h_{3} z^{5}+h_{3}^{2} z^{6}
$$

Using formulae (18)-(25), the final RMS energy spread of the bunch is calculated through integration, and shown in formula (41).

$$
\sigma_{\delta}^{2}=a^{2} \sigma_{\delta i}^{2}+h_{1}^{2} \cdot \sigma_{z, i}{ }^{2}+3 \cdot\left(2 h_{1} h_{3}+h_{2}^{2}\right) \cdot \sigma_{z, i}{ }^{4}+15 \cdot h_{3}^{2} \cdot \sigma_{z, i}{ }^{6}
$$

where $h_{2}$ denotes the second order RF chirp, $h_{3}$ the third order RF chirp. 

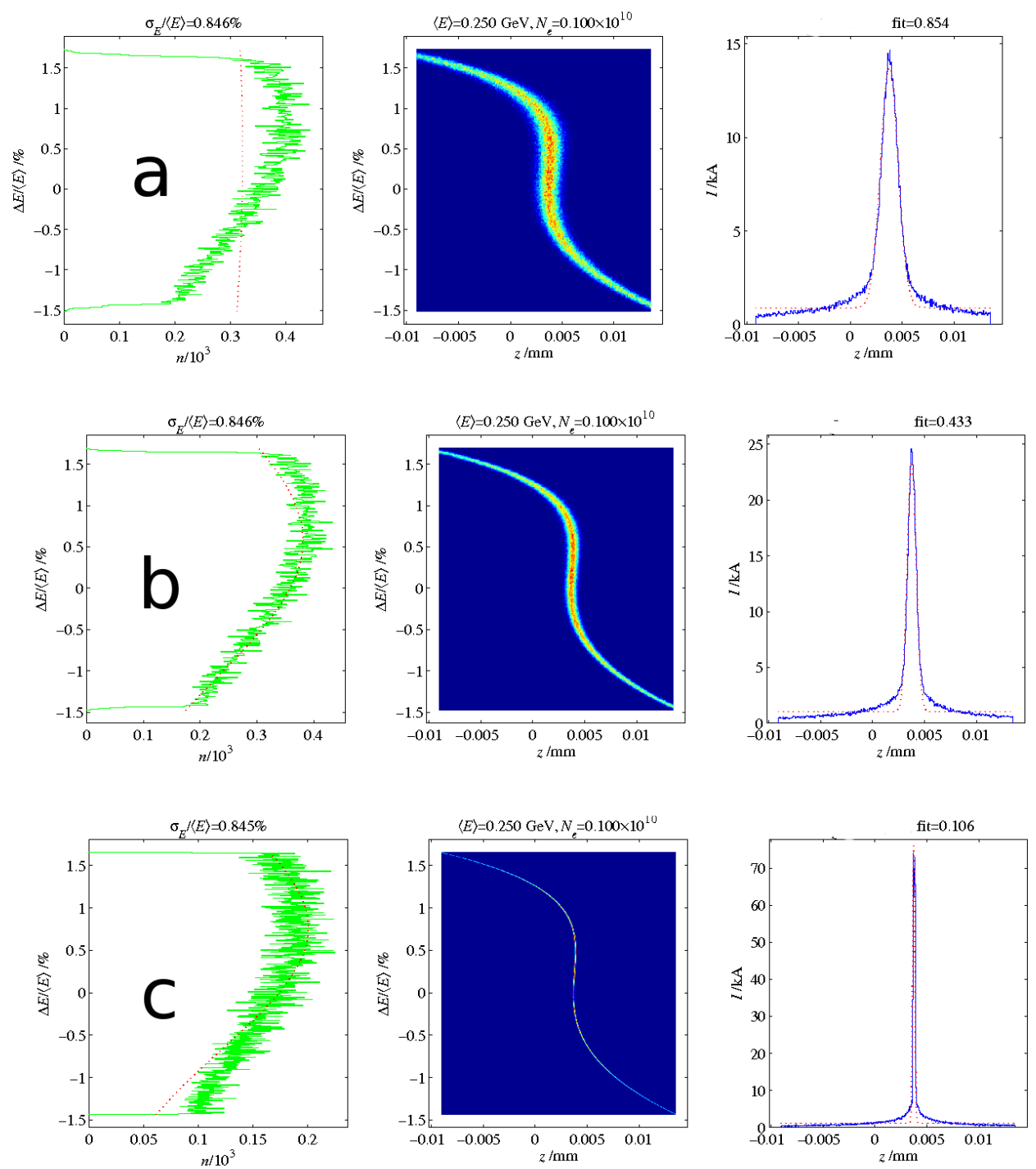

Figure 3: LiTrack simulation results, at the exit of the bunch compressor and with a beam energy of $250 \mathrm{MeV}$. (a): initial un-correlated energy spread of $0.2 \%$; (b): initial un-correlated energy spread of $0.1 \%$; (c): initial un-correlated energy spread of $0.01 \%$.

\section{Parameter selection}

In this section, the formulae derived in the above sections are applied to evaluate the parameter relations, and finally to choose a group of possible parameters for the proposed x-band FEL driver.

\subsection{Bunch length and peak current}

At the exit of the photo injector ( $5 \mathrm{MeV}$ ), a realistic RMS bunch length can be as short as $300 \mu m$ (bunch charge $250 p C)$. The initial bunch length before compression is assumed to be $300 \mu m$ with a bunch charge of $250 p C$. One can achieve a shorter bunch length from the injector with less charge which is discussed in the following sections. 

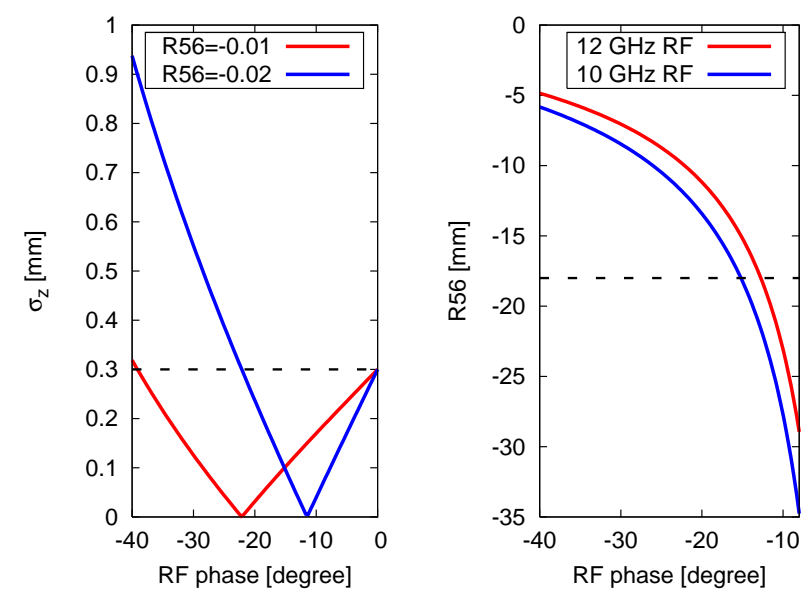

Figure 4: Left: final RMS bunch length as a function of the RF phase, only first order term is considered. Right: required $R_{56}$ as a function of the $\mathrm{RF}$ phase, to fulfill the minimum bunch length condition.
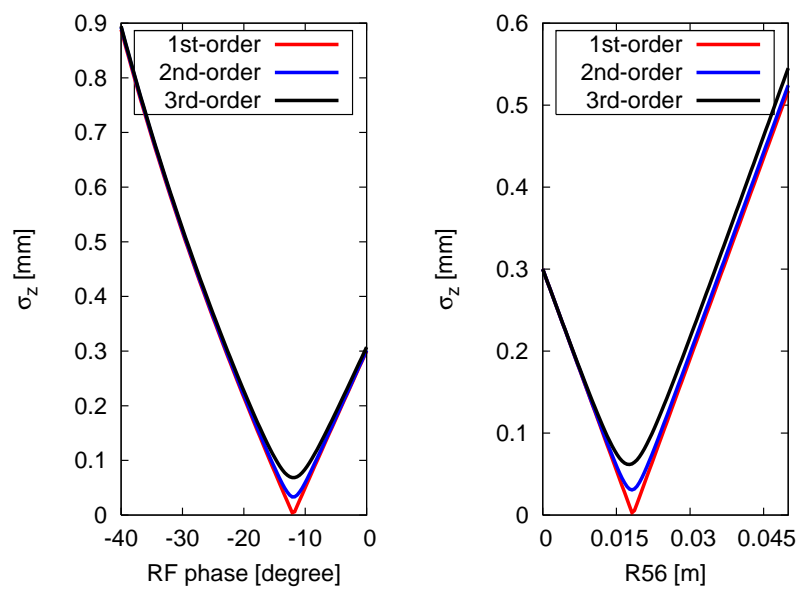

Figure 5: Left: final RMS bunch length as a function of the RF phase, consider up to first, second and third order terms $\left(R_{56}=20 \mathrm{~mm}\right)$. Right: final RMS bunch length as a function of $R_{56}$ (with a fixed RF phase of 13 degree), consider up to first, second and third order terms.

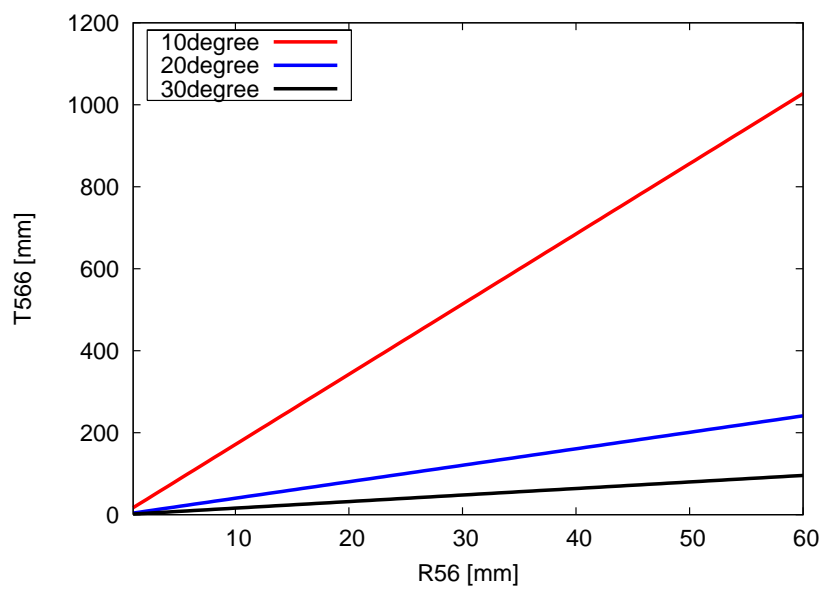

Figure 6: $T_{566}$ as a function of $R_{56}$, to achieve the minimum bunch length. Three different RF phase (10, 20 and 30 degree) is considered. 
After bunch compression, regardless of the bunch head and tail, the central part can be treated to have a normal distribution of the form

$$
f(x)=\frac{1}{2 \pi \sigma} \cdot \exp \left[-\frac{\left(x-x_{0}\right)^{2}}{2 \sigma^{2}}\right]
$$

where $\sigma$ denotes the standard deviation.

The relationship between the full width at half maximum (FWHM) and the RMS value of the standard deviation is

$$
F W H M=2 \sqrt{2 \ln 2} \cdot \sigma \approx 2.35482 \sigma
$$

As discussed above, the FEL prefers a higher peak current. For soft X-ray FEL the peak current is required to be above $1 k A$, and for hard X-ray it is $3 k A$. The peak current can be calculated, or estimated for some kind of bunch distributions, as shown in formula (44).

$$
I_{\text {peak }}=\frac{Q}{2 \sqrt{2 \ln 2} \cdot \sigma / c}
$$

where $I_{\text {peak }}$ denotes the peak current, $Q$ total bunch charge in $C, \sigma$ the standard deviation (RMS bunch length), and $c$ speed of light.

For an initial bunch length of $300 \mu \mathrm{m}$ and a bunch charge of $250 \mathrm{pC}$, the peak current is $0.1 \mathrm{kA}$, according to formula (44). That means one needs to compress the bunch length by 30 times, in order to achieve a peak current of $3 k A$. It is very challenging to do so by a single stage bunch compressor and at a beam energy of $250 \mathrm{MeV}$.

\section{2. $R_{56}, T_{566}$ and RF phase}

Condider only first order term in formula (29), and treat the bunch length as a function of the RF phase, the final bunch length with two different $R_{56}$ is shown in Figure 4 (left). It is observed that for different $R_{56}$, the bunch length has a minimum at different RF phase. The minimum bunch length is dependent on the initial un-correlated energy spread, $\sigma_{z}=a R_{56} \sigma_{\delta i}$, as shown in formula (29). At the same time, to fulfill the minimum bunch length condition, one needs to take $1+h_{1} R_{56}=0$, if only first order term is taken into account. This ends up with a relation between $R_{56}$ and the RF phase $\phi$, as shown in Figure 4 (right), for two different RF frequencies. One can observe that for a same $R_{56}$, the required RF phase is smaller with a RF frequency of $12 G H z$ than with $10 G H z$. However, the difference is small.

Use formula (29), the final bunch length is plotted in Figure 5 (left), again as a function of the RF phase. It is observed that the minimum bunch length is much longer with higher order effects taken into account (for chicane optics, $T_{566}=-1.5 \cdot R_{56}, U_{5666}=2 \cdot R_{56}$ ). A similar plot is shown in Figure 5 (right), where the RF phase is fixed to be 13 degree and the final bunch length is compared at different $R_{56}$. Here it is observed that for the case with a chicane as bunch compressor, the higher order terms $T_{566}$ and $U_{5666}$ always make the final bunch length longer, if the initial longitudinal phase space is not linearized. With a harmonic RF linearization, the higher order effects can be minimized and one can resume a short bunch length. As mentioned above, it is much more difficult to develop and operate a harmonic RF for x-band RF, so an alternative way is to use an optics with specified $T_{566}$ and $U_{5666}$ to compensate the RF curvature.

In Figure 6, the second order longitudinal dispersion $T_{566}$ is plotted as a function of $R_{56}$ according to formula (36), with three different RF phase. It is observed that for a smaller RF phase, the slope of the curve is larger. The detailed discussion and derivation on optics and higher order dispersions will be presented in another paper [6].

As discussed in the next section, here a RF phase of 13 degree is chosen, which requires a $R_{56}$ of $18 \mathrm{~mm}$ and a $T_{566}$ of $160 \mathrm{~mm}$, to achieve the minimum final bunch length. 

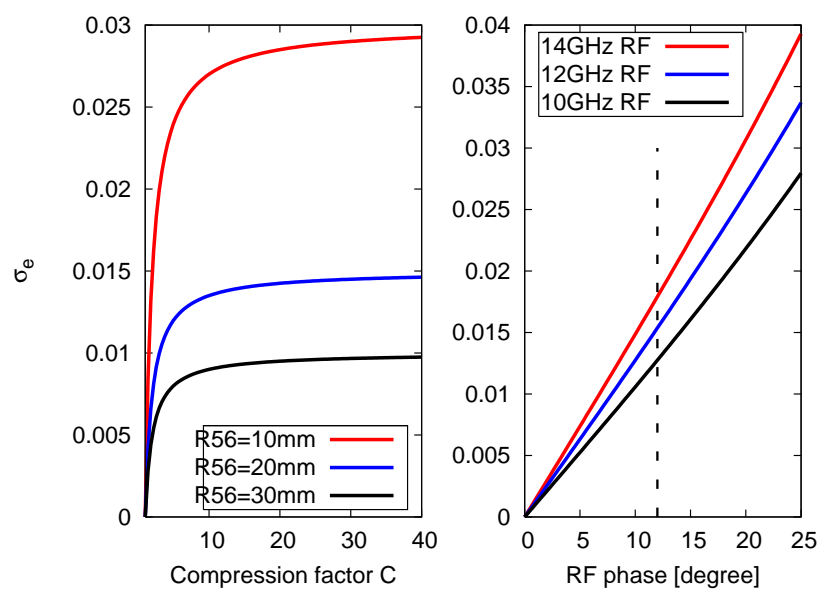

Figure 7: Left: final energy spread as a function of the linear compression factor $C$; Right: final energy spread as a function of the RF phase, for three different RF frequencies.
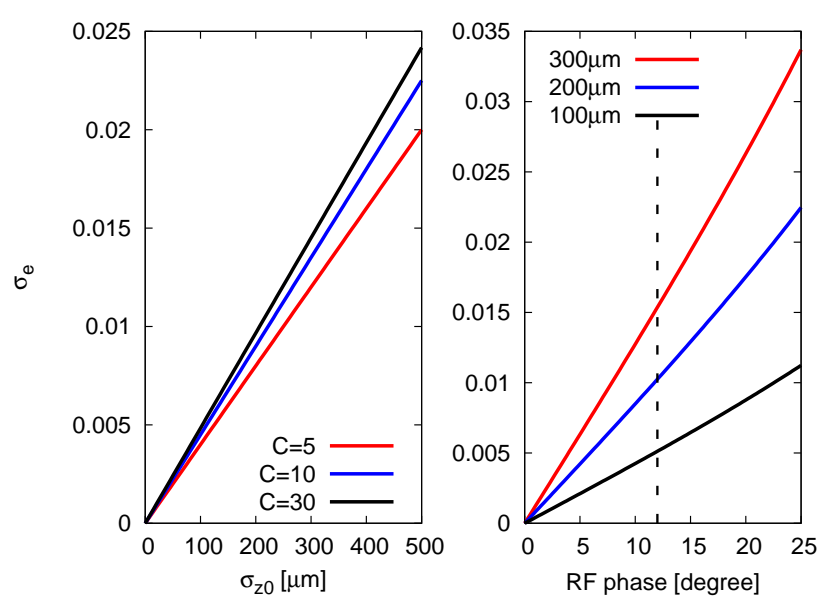

Figure 8: Left: final energy spread as a function of the initial bunch length, for three different compression factor $C$; Right: final energy spread as a function of the RF phase, for three different initial bunch length.

\subsection{Final energy spread}

The initial un-correlated energy spread is small, which is $10 \mathrm{keV}$ at a beam energy of $5 \mathrm{MeV}\left(\sigma_{e 0}=0.2 \%\right)$. After acceleration to a beam energy of $250 \mathrm{MeV}$, the un-correlated energy spread is further damped by 25 times.

The main contribution to the final energy spread is from the RF chirp. As discussed above, the key point of magnetic bunch compression is to generate a bunch with z-correlated energy, and then let it pass by a dispersive region. A large energy spread is also generated on the bunch when the chirp is established during off-crest RF acceleration process. Compared with LCLS, here a RF frequency of $12 \mathrm{GHz}$ (x-band) is applied, instead of $3 G H z$ (S-band). At the same time, the initial bunch length is assumed to be $300 \mu m$ with a bunch charge of $250 p C$. For LCLS the initial bunch length from injector is $600 \mu m$ with a bunch charge of $250 p C$. Consider both RF wave length and initial bunch length, the energy spread for the proposed x-band FEL driver is 2 times of LCLS case.

The linear compression factor is defined as

$$
C=\frac{1}{1+h_{1} R_{56}}
$$

where $h_{1}$ denotes the linear (first order) chirp, $R_{56}$ the first order longitudinal dispersion. 

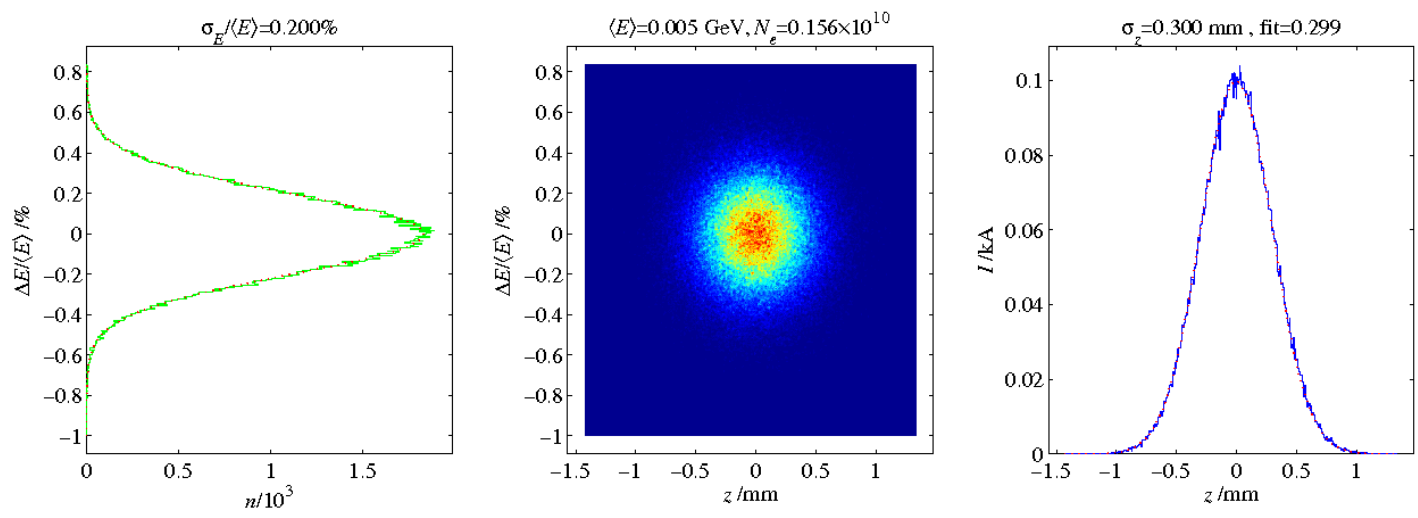

Figure 9: Initial longitudinal distribution and phase space.

According to formulae (41) and (45), the final energy spread is plotted as a function of the linear compression factor $C$, as shown in Figure 7 (left). For a compression factor of 10 and above, the 'intrinsic' energy spread tends to be similar. A higher RF phase is needed for a smaller $R_{56}$, and the final energy spread is larger in that case, as shown by the red curve in Figure 7 (left). As shown in Figure 7 (right), the final energy spread is plotted as a function of the RF phase, again according to formula (41). One observes that with a lower RF frequency of $10 G H z$, the energy spread is smaller. However, one finds that the difference on RF frequency is not so large.

The plot in Figure 8 (left) indicates that the final energy spread has a linear relation with the initial bunch length, and again for different compression factor $(C=5, C=10$ and $C=30)$ the final energy spread is similar with a same initial bunch length. For a bunch compression of 30 times and an initial bunch length of $300 \mu m$, the final RMS energy spread is $1.7 \%$. A large energy spread may make the transport of the electron bunch through the bunch compressor more difficult, due to dispersive and chromatic effects. An initial bunch length of $200 \mu m$ only results in a RMS energy spread of $1.0 \%$, as illustrated in Figure 8 (right),

\section{Longitudinal 1-D simulation with LiTrack}

In this section, the longitudinal one dimentional simulation of the proposed $\mathrm{x}$-band FEL driver is implemented in LiTrack [7], and the simulation results are presented.

\subsection{Optimal compression}

For the optimal compression case, one tries to minimize the final bunch length by choosing proper $R_{56}, T_{566}$ and $U_{5666}$, according to formulae (35)-(37). Here we recall the parameters that have been chosen for the x-band FEL driver. The initial bunch from the photo injector has a RMS bunch length of $300 \mu m$, and a bunch charge of $250 p C$. The electron population per bunch is $0.156 \times 10^{10}$, and the RMS energy spread is $0.2 \%$. The peak current is $0.1 k A$, as shown in Figure 9.

The RF frequency is chosen to be $12 G H z$ which is slightly different with the one being operated at SLAC now. A $\mathrm{RF}$ phase of 13 degree is used, in order to get a smaller final energy spread. It is noted that one degree of $\mathrm{x}$-band RF (12GHz) covers roughly $70 \mu m$ range in length. The choice of 13 degree makes the head (or tail) of the bunch (at $3 \sigma_{z}$ ) right on $\mathrm{RF}$ crest. The longitudinal phase space is shown in Figure 10, for a bunch which has just passed by the RF accleration part. The chirp is shown in the middle plot of Figure 10. One notes that the RF phase here 

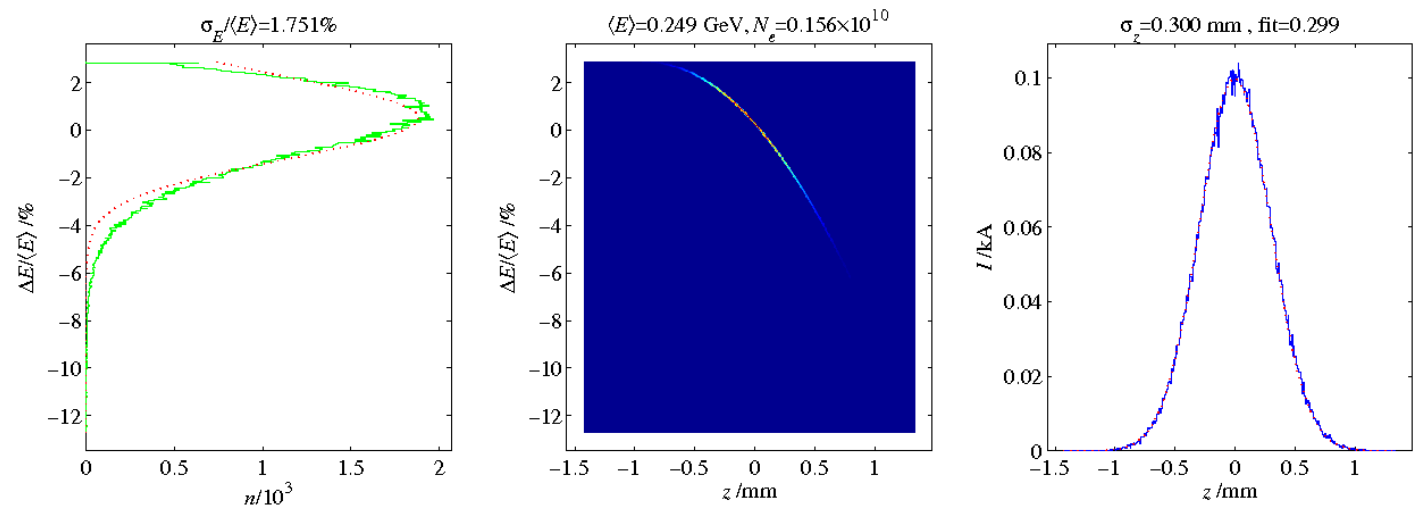

Figure 10: Longitudinal phase space and distribution after RF chirp.
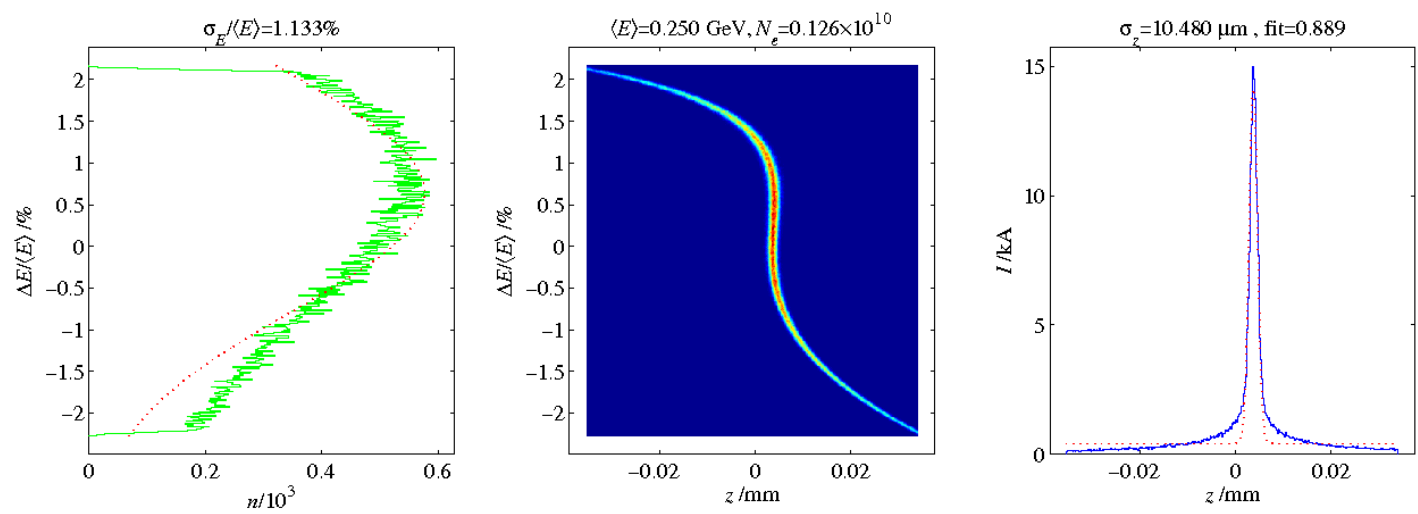

Figure 11: Longitudinal phase space and distribution after dispersive region, optimal compression up to 2nd-order (considering only $\left.T_{566}\right)$.

is positive, while the RF phase for LCLS is chosen to be negative (assuming zero phase to be on crest), due to the opposite sign of $R_{56}$ being applied.

According to formula (35), the first order dispersion $R_{56}$ needed from the dispersive region is $17.6 \mathrm{~mm}$. One finds the required higher order dispersions are $T_{566}=168 \mathrm{~mm}$ and $U_{5666}=1 \mathrm{~m}$, according to formulae (36) and (37), respectively.

In LiTrack, a simple model is established, where the RF chirp is represented by a RF element type '11' and the dispersive region represented by a bunch-compressor element type ' 6 '. Collimators (the elements with type '27' and type ' 37 ' in LiTrack) are used to clean the particles which has an absolute energy offset larger than $2 \%$.

If the bunch compressor is designed to provide up to second order correction (with $R_{56}=17.6 \mathrm{~mm}$ and $T_{566}=$ $168 \mathrm{~mm}$ ), the final longitudinal bunch phase space is shown in Figure 11. In this case, the final bunch length is $0.889 \mu \mathrm{m}$ from FWHM fit, and a peak current of $15 k A$ is achieved. If the third order dispersion of the bunch compressor $U_{5666}$ is also corrected according to formula (37), one has $R_{56}=17.6 \mathrm{~mm}, T_{566}=168 \mathrm{~mm}$ and $U_{5666}=1 \mathrm{~m}$. In that case, the final bunch length is slightly shorter $\left(\sigma_{z}=0.861 \mu m\right)$, compared with $0.889 \mu m$ of the previous case. A similar longitudinal phase space is shown in Figure 12. 

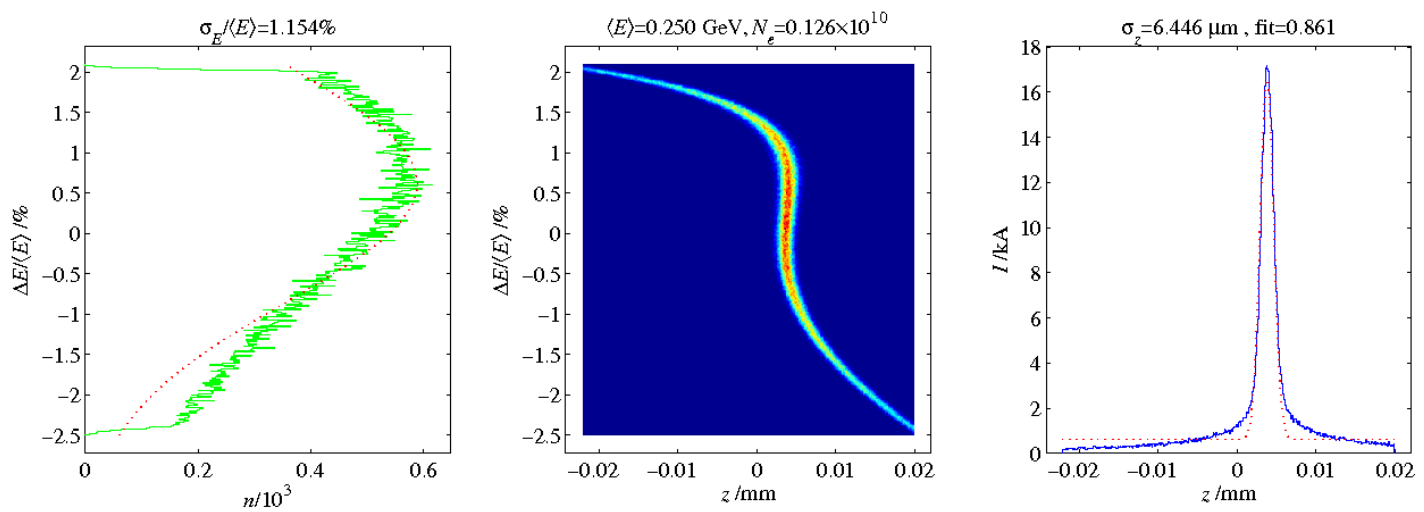

Figure 12: Longitudinal phase space and distribution after dispersive region, optimal compression up to 3rd-order (considering both $T_{566}$ and $\left.U_{5666}\right)$.
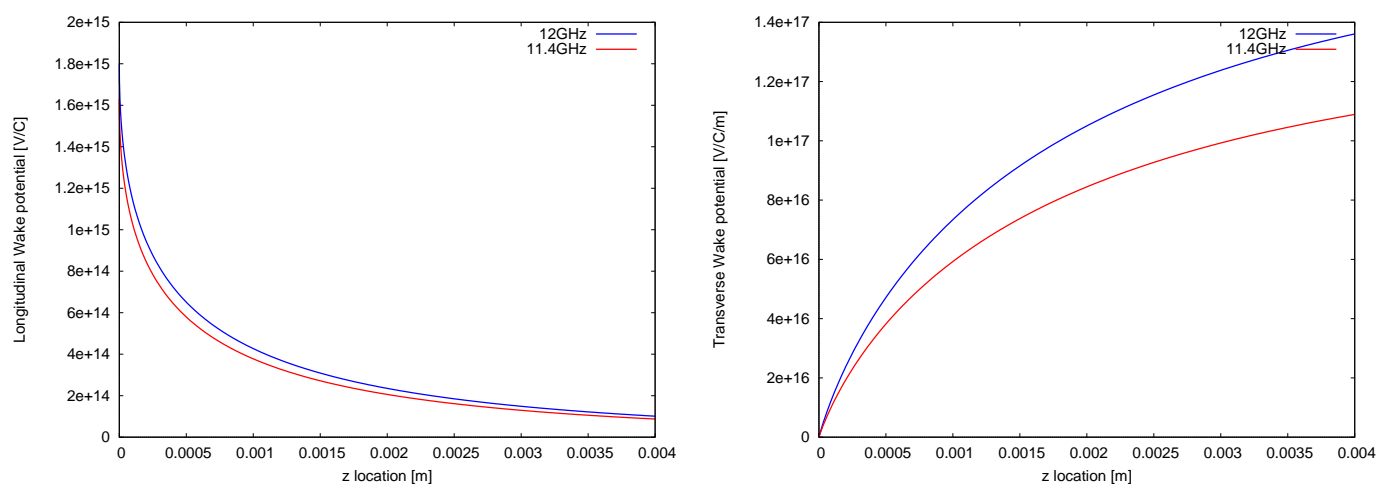

Figure 13: Single bunch longitudinal wake potential (left) and transverse wake potential (right), from x-band acceleration structure.

\subsection{Wake field effects}

The wake field effects can be more severe for x-band structures, due to their smaller apertures, in comparison with lower frequency RF structures such as S-band.

An analytical formula of the longitudinal wake potential (per unit length, or meter) for a point charge is listed below, derived by K. Bane [8]. The longitudinal wake field can then be integrated, using this wake potential plus the real bunch distribution and bunch charge, over the length of the RF section.

$$
W_{L}(s) \approx \frac{Z_{0} c}{\pi a^{2}} \phi(s) \exp \left(-\sqrt{\frac{s}{s_{00}}}\right)
$$

where $Z_{0}$ denotes longitudinal impedance of the x-band structure (vacuum impedance), $c$ speed of light, $a$ iris radius, $\phi(s)$ a step function $(\phi(s)=1$ for $s>0$, and $\phi(s)=0$ for $s<0)$.

$$
s_{00}=\frac{g}{8} \cdot\left(\frac{a}{a(g / L) \cdot L}\right)
$$

where $g$ denotes the gap, $L \mathrm{RF}$ period, and $b$ cavity radius.

Similarly, an asymptotic short-range solution is achieved with the fitting method, and the single bunch transverse wake potential is then expressed as in formula (48) [8]. 

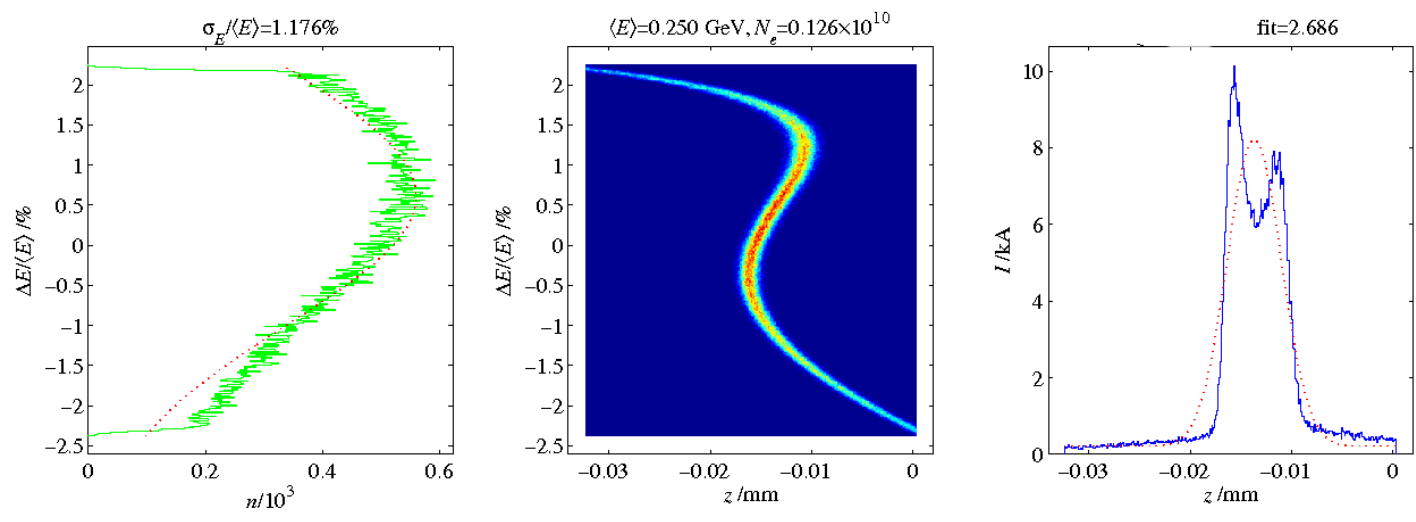

Figure 14: Phase space and distribution after dispersive region (with single bunch longitudinal wake field in the X-band acceleration region), optimal compression up to 3rd-order $\left(U_{5666}\right)$. It is an over-compression case in effective.
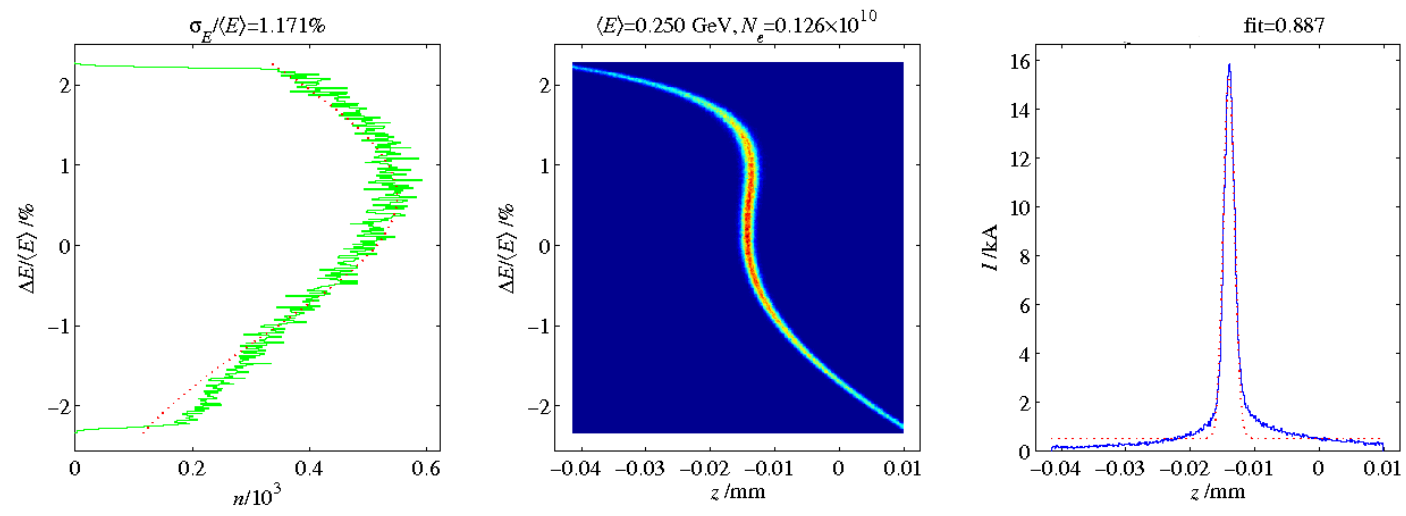

Figure 15: Phase space and distribution after dispersive region (with single bunch longitudinal wake field in the X-band acceleration region and decreased $\left.R_{56}\right)$, optimal compression up to 3 rd-order $\left(U_{5666}\right)$.

$$
\begin{gathered}
W_{x}(s) \approx \frac{4 Z_{0} c s_{0}}{\pi a^{4}} \phi(s)\left[1-\left(1+\sqrt{\frac{s}{s_{0}}}\right) \cdot \exp \left(-\sqrt{\frac{s}{s_{0}}}\right)\right] \\
s_{0}=0.169 \cdot \frac{a^{1.79} g^{0.38}}{L^{1.17}}
\end{gathered}
$$

The single bunch longitudinal and transverse wake potential are plotted in Figure 13 (left) and (right), respectively.

The longitudinal single bunch wake field applies a chirp (z-correlated energy modulation) which has the same sign as the RF chirp in our case. In comparison, for LCLS the wake field longitudinal chirp always has an opposite sign as their RF chirp. In order to compensate this effect, either the RF phase or the linear longitudinal dispersion $R_{56}$ needs to be adjusted (decreased) in our case. In Figure 14, the longitudinal phase space is shown for the designed optimal compression case, which is destroyed by the additional chirp of the longitudinal wake field. The final bunch length is increased to $2.7 \mu \mathrm{m}$ accordingly. As stated before, the minimum bunch length condition can be resumed, by applying a smaller $R_{56}$ in the bunch compressor, as shown in Figure 15. 


\subsection{Realistic case as FEL driver: three options}

A FEL may have different operating modes which makes use of different bunch charge and bunch intensity. For the FEL driver studied here, it is already mentioned that a smaller final energy spread prefers a shorter initial bunch length, which means the bunch charge need to be lower. For the normal LCLS operation, the single bunch charge is either $20 p C$ or $250 p C$.

With the aim of producing hard X-ray, three options are studied here, each with a specified initial bunch length and bunch charge from the photo injector. For a bunch charge of $20 p C$ (option 'A'), $120 p C$ (option 'B') and $250 p C$ (option 'C'), the initial bunch length is $\sigma_{z}=80 \mu \mathrm{m}, 150 \mu \mathrm{m}$ and $300 \mu \mathrm{m}$, respectively.

For all three options, we manage to achieve a peak (or uniform) current of $3 k A$ with an over-compressed bunch, in a single stage bunch compression at $250 \mathrm{MeV}$, as shown in Figure 16. The over-compressed bunch is then further accelerated to $6 \mathrm{GeV}$ for FEL applications afterwards. Longitudinal wake field in the second linac is used to de-chirp the bunch and generate a 'flat' bunch which has a uniform energy. For the options ' $\mathrm{B}$ ' and ' $\mathrm{C}$ ', a proper RF phase is also used as the wake field effects is not enough to remove the initial chirp. The longitudinal phase space at a beam energy of $6 \mathrm{GeV}$ is shown in Figure 17.

\section{Conclusion}

In this paper, a single stage bunch compressor plus $\mathrm{x}$-band RF is proposed as a FEL driver. Analytical formulae are derived to evaluate the relations between different beam, RF and bunch-compressor parameters. 1-D simulation in LiTrack demonstrates that an electron beam (to generate hard X-ray FEL) with a peak current of $3 k A$ can be generated by this system. Optics design for the bunch compressor, and a full 3-D simulation in Elegant is underway, to study the impact on the transverse emittance etc..

\section{Acknowledgement}

The authors would like to thank M. Woodley, A. Chao, P. Emma, Z. Huang, C. Adolphsen et al. for helpful discussions.

This work was supported by the DOE under Contract DE-AC02-76SF00515.

\section{References}

[1] J. Madey, J. Appl. Phys. 42, 1906 (1971).

[2] D. A. G. Deacon, L. R. Elias, J. M. J. Madey, G. J. Ramian, H. A. Schwettman, and T. I. Smith, Phys. Rev. Lett. 38, 892 (1977).

[3] J. Rossbach, in Proceedings of the 2006 European Particle Accelerator Conference, p. 34 (2006).

[4] Linac Coherent Light Source Conceptual Design Report, SLAC-R-593, SLAC (2002); P. Emma et al., Nature Photonics 4, 641-647 (2010)

[5] TESLA Technical Design Report, TESLA FEL 2002-09, DESY, 2002; SPring-8 Compact SASE Source Conceptual Design Report, http://www-xfel.spring8.or.jp (2005); I. S. Ko, in Proceedings of the 2005 Free Electron Laser Conference (Stanford, CA, USA, 2005), p. 216.

[6] Yipeng Sun et al., to be published.

[7] K.L.F. Bane and P. Emma, "Litrack: A Fast Longitudinal Phase Space Tracking Code with Graphical User Interface", Proceedings of the 2005 Particle Accelerator Conference, 16-20 May 2005, pp. 4266-4268 (2005).

[8] K.L.F. Bane, SLAC pub-9663 (2003). 

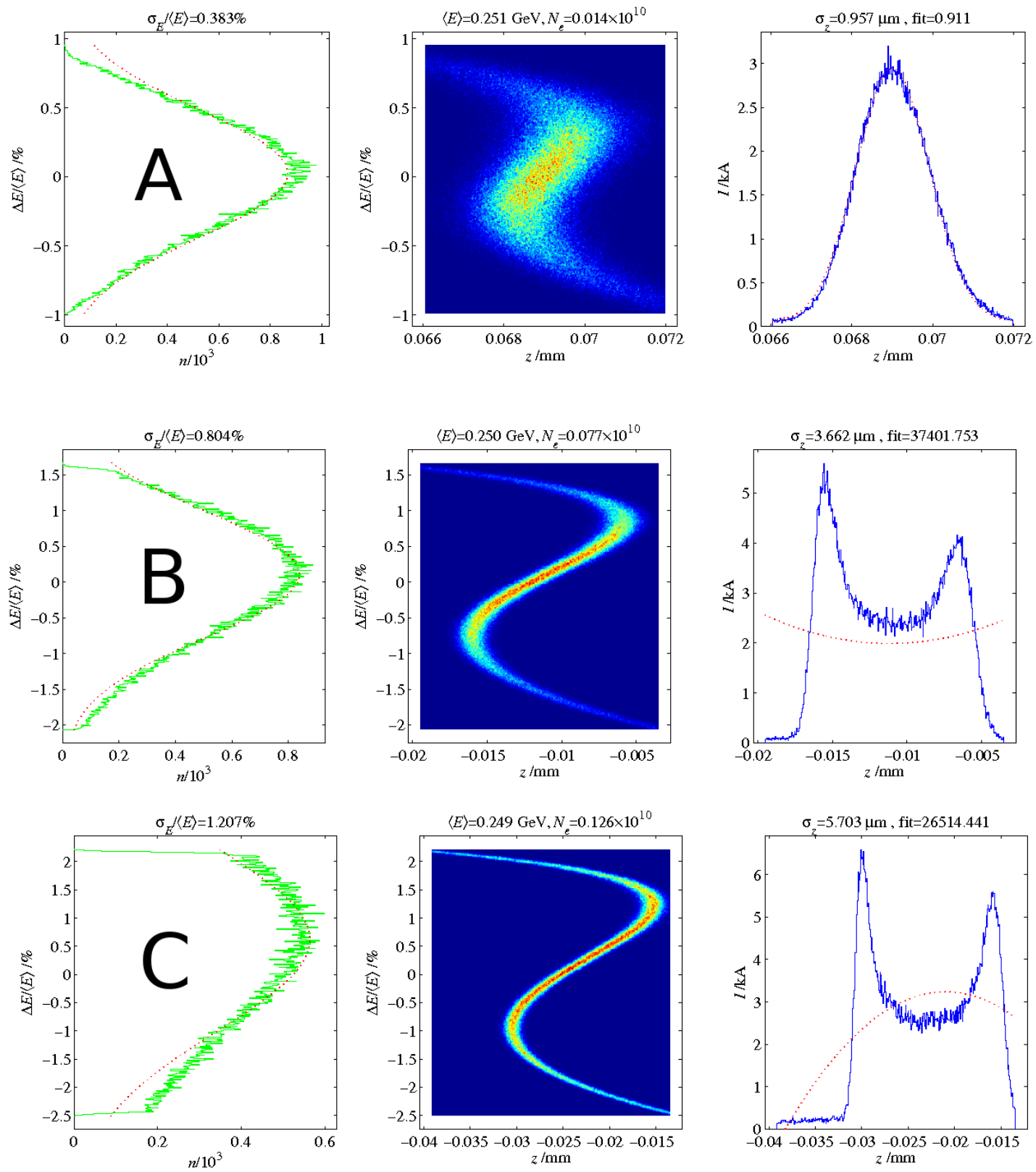

Figure 16: LiTrack simulation results, at the exit of the bunch compressor and with a beam energy of $250 \mathrm{MeV}$. (A): bunch charge of $20 \mathrm{pC}$, initial bunch length of $80 \mu \mathrm{m}$; (B): bunch charge of $120 \mathrm{pC}$, initial bunch length of $150 \mu \mathrm{m}$; (C): bunch charge of $250 \mathrm{pC}$, initial bunch length of $300 \mu \mathrm{m}$.

\section{: Appendix ALinearization by using harmonic RF}

To linearize the RF curvature on the energy chirp, a harmonic RF section is required which has a higher frequency. The energy offset of any particle after passing by these two RF section is expressed in the following formula, where the third term on the right side is from the harmonic RF.

$$
\delta(z)=\delta_{i} \frac{E_{i 0}}{E_{f 0}}+\frac{e V_{0} \cos \left(\phi+k z_{i}\right)}{E_{f 0}}+\frac{e V_{h} \cos \left(\phi_{h}+k_{h} z_{i}\right)}{E_{f 1}}
$$



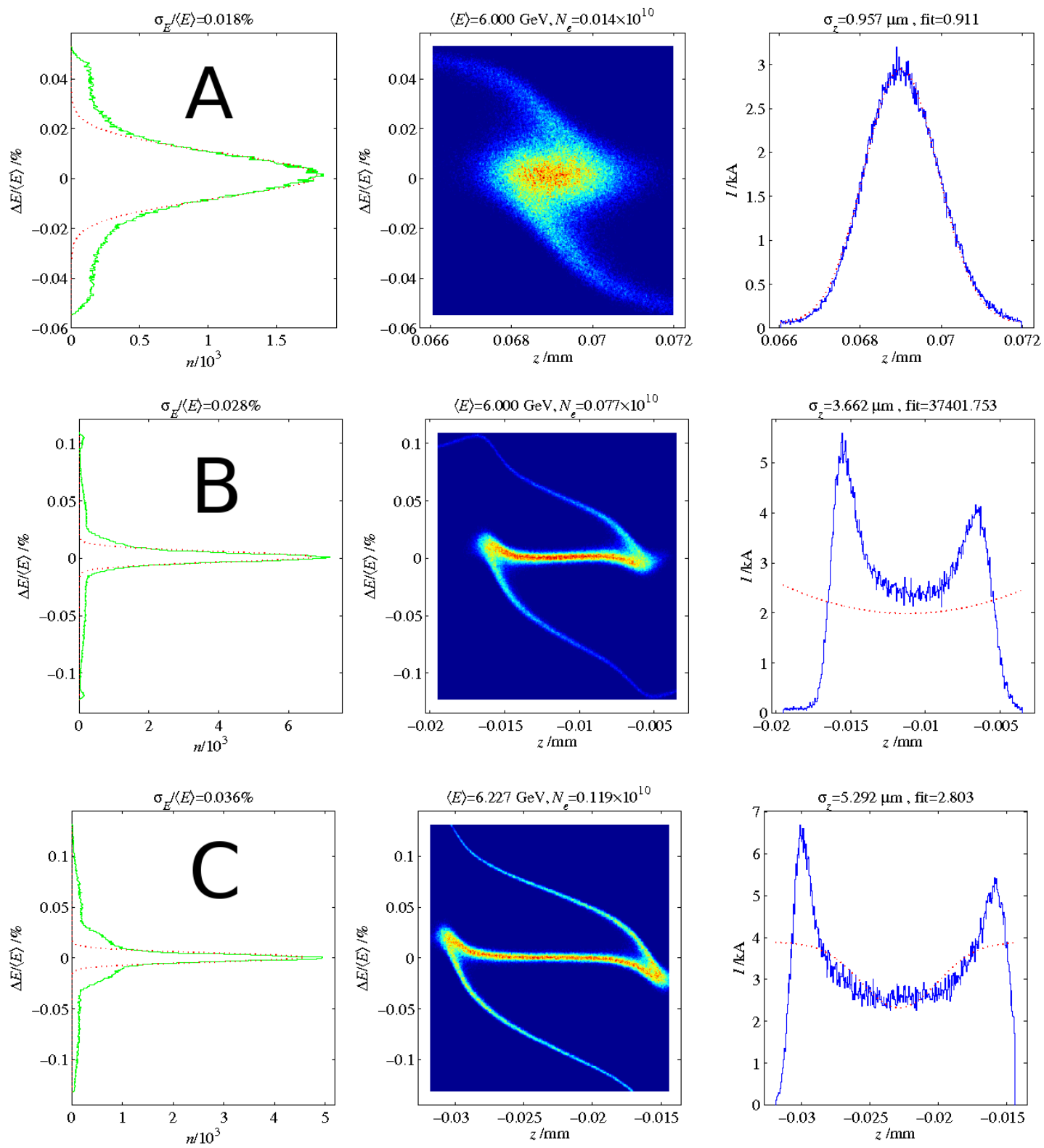

Figure 17: LiTrack simulation results, at the end of the linac and with a beam energy of $6 \mathrm{GeV}$. (A): bunch charge of $20 \mathrm{pC}$, initial bunch length of $80 \mu m$; (B): bunch charge of $120 \mathrm{pC}$, initial bunch length of $150 \mu \mathrm{m}$; (C): bunch charge of $250 \mathrm{pC}$, initial bunch length of $300 \mu \mathrm{m}$. De-chirp is done with longitudinal wake field and a proper RF phase.

where $V_{h}$ denotes the RF voltage of harmonic RF, $E_{f 1}$ central energy after $\mathrm{RF}$ acceleration and deceleration, $V_{h}$ harmonic RF voltage, $\phi_{h}$ harmonic RF phase, $k_{h}=\frac{2 \pi f_{h}}{c}$ the harmonic RF wave number, $f_{h}$ the RF frequency and $c$ speed of light.

We recall the polynomial expression of the z-correlated energy offset, after passing by the first RF section, as shown below.

$$
\delta(z)=h_{1 s} z+h_{2 s} z^{2}+h_{3 s} z^{3}+\ldots
$$


where $h_{1 s}$ denotes the first order chirp, $h_{2 s}$ second order chirp, and $h_{3 s}$ third order chirp (the name 's' reflects that it is assumed to be s-band as for the normal case).

$$
\begin{gathered}
h_{1 s}=-\frac{k e V_{0} \sin \phi}{E_{f 0}} \\
h_{2 s}=-\frac{k^{2} e V_{0} \cos \phi}{2 E_{f 0}} \\
h_{3 s}=\frac{k^{3} e V_{0} \sin \phi}{6 E_{f 0}}
\end{gathered}
$$

where $e$ denotes the electron charge.

Similarly, for the harmonic RF, one has

$$
\delta_{h}(z)=h_{1 h} z+h_{2 h} z^{2}+h_{3 h} z^{3}+\ldots
$$

where $h_{1 h}$ denotes the first order harmonic chirp, $h_{2 h}$ second order harmonic chirp, and $h_{3 h}$ third order harmonic chirp.

Up to third order, it is easy to write down the overall energy chirp, after passing by these two RF sections, as shown in the formulae below.

$$
\begin{gathered}
h_{1}=h_{1 s}+h_{1 h}=-\frac{k e V_{0} \sin \phi}{E_{f 0}}-\frac{k_{h} e V_{h} \sin \phi_{h}}{E_{f 1}} \\
h_{2}=h_{2 s}+h_{2 h}=-\frac{k^{2} e V_{0} \cos \phi}{2 E_{f 0}}-\frac{k_{h}^{2} e V_{h} \cos \phi_{h}}{2 E_{f 1}} \\
h_{3}=h_{3 s}+h_{3 h}=\frac{k^{3} e V_{0} \sin \phi}{6 E_{f 0}}+\frac{k_{h}^{3} e V_{h} \sin \phi_{h}}{6 E_{f 1}}
\end{gathered}
$$

One observes that to generate a linearized energy chirp up to second order, the expression in formula (A8) need to equal zero. Taking advantage of formula (A10), the requirement on the harmonic RF could be solved and presented in formula (A11) below.

$$
E_{f 1}=E_{f 0}+V_{h} \cos \phi_{h}
$$

where $E_{f 0}$ denotes the beam central energy after the first RF section, $E_{f 1}$ the central energy after harmonic RF section.

$$
V_{h} \cos \phi_{h}\left(E_{f 0} \cdot k_{h}^{2}+k^{2} \cdot V_{0} \cos \phi\right)=-E_{f 0} k^{2} \cdot V_{0} \cos \phi
$$

It is straightforward to observe that one needs a decelerating harmonic RF phase $\phi_{h}$, given an accelerating $\mathrm{RF}$ phase $\phi$. Similarly solve the zero condition for formula (A9), one finds that the third order linearization condition reads

$$
E_{f 0} k_{h}^{3} V_{h} \sin \phi_{h}+k^{3} \cdot V_{0} \sin \phi \cdot V_{h} \cos \phi_{h}=-E_{f 0} k^{3} \cdot V_{0} \sin \phi
$$


Insert formula (A13) into formula (A11), one finds a simpler relation as shown in formula (A14), for second order linearization.

$$
V_{0} \cos \phi=E_{f 0}-E_{i 0}
$$

$$
V_{h} \cos \phi_{h}\left(E_{f 0} \cdot k_{h}^{2}+k^{2} \cdot\left(E_{f 0}-E_{i 0}\right)\right)=-E_{f 0} k^{2} \cdot\left(E_{f 0}-E_{i 0}\right)
$$

